\title{
ТЕХНОЛОГИЯ ИНТЕРФЕЙСА МОЗГ-КОМПЬЮТЕР КАК КОНТРОЛИРУЕМЫЙ ИДЕОМОТОРНЫЙ ТРЕНИНГ В РЕАБИЛИТАЦИИ БОЛЬНЫХ ПОСЛЕ ИНСУЛЬТА
}

\author{
Ю. В. Бушкова ${ }^{1 凶}$, Г. Е. Иванова \\ 1 Федеральный центр цереброваскулярной патологии и инсульта, Москва, Россия \\ 2 Российский национальный исследовательский университет имени Н. И. Пирогова, Москва, Россия \\ ${ }^{3}$ Институт высшей нервной деятельности и нейросизиологии, Москва, Россия
}

\begin{abstract}
Восстановление функции руки у пациентов после инсульта является приоритетным направлением в нейрореабилитации. Развитие технологии интерфейса мозг-компьютер-экзоскелет кисти (ИМКЭ) качественно улучшило реабилитацию в этом направлении. Целью данного исследования было изучить факторы, влияющие на двигательное восстановление верхней конечности у пациентов после инсульта на фоне применения технологии ИМКЭ. Исследовали 24 пациента (14 мужчин, 10 женщин) в возрасте от 51 до 62 лет с единичным очагом инсультной этиологии, супратенториальной локализации. В 11 (45,6\%) случаях левополушарное поражение, в 13 (54,4\%) случаях — правополушарное. Давность инсульта - 4,0 (3,0; 12,0) месяца Медиана МоСА - 25,0 (23,0; 27,0). Курс занятий ИМКЭ включал 9,5 (8,0; 10,0) процедур. Выявлена значимая умеренная корреляция между успешностью моторного представления (MIQ-RS) и эффективностью взаимодействия пациентов с ИМКЭ кисти. Пациенты с высокими показателями МIQ-RS 47,5 (32,0; 54,0) достоверно лучше взаимодействовали с ИМКЭ 63,0 (54,0; 67,0), $R=0,67$ ( $<$ 0,05). У пациентов с высокими показателями MIQ-RS динамика двигательного восстановления была более выраженной: медиана $\Delta$ Fugl-Meyer Assessment (FMA) составила 14 (8,0; 16,0$)$ баллов против группы пациентов с низкими показателями MIQ-RS, $\triangle \mathrm{FMA}$ - 10 (6,0; 13,0), но при этом не достигла статистически значимого уровня. Таким образом, выявлена взаимосвязь между способностью пациентов к моторному представлению (MIQ-RS) и эффективностью взаимодействия пациентов с ИМКЭ. Для выявления влияния этих факторов на динамику двигательного восстановления руки, вероятно, требуется продолжить исследование с большей выборкой.
\end{abstract}

Ключевые слова: инсульт, нейрореабилитация, интерфейс мозг-компьютер, идеомоторная тренировка

Финансирование: исследование поддержано Российским фондом фундаментальных исследований (грант № 16-29-08247 офи_м).

Информация о вкладе авторов: Ю. В. Бушкова - планирование исследования, анализ литературы, сбор, анализ и интерпретация данных, подготовка черновика рукописи; Г. Е. Иванова — планирование исследования, анализ литературы, интерпретация данных, подготовка рукописи; Л. В. Стаховская планирование исследования, интерпретация данных, подготовка рукописи; А. А. Фролов - подготовка рукописи.

Соблюдение этических стандартов: исследование одобрено этическим комитетом Научного центра неврологии (протокол № $12 / 14$ от 10 декабря 2014 г.). Протокол исследования іMove зарегистрирован в международном реестре клинических исследований Национального института здоровья США Clinical Trials. gov (identifier: NCT02325947). Все пациенты подписали добровольное информированное согласие на участие в исследовании.

$\checkmark$ Для корреспонденции: Юлия Владимировна Бушкова

ул. Островитянова, д. 1, стр. 10, г. Москва, 117342; Julijabush777@gmail.com

Статья получена: 12.11.2019 Статья принята к печати: 24.11.2019 Опубликована онлайн: 07.12.2019

DOI: $10.24075 /$ vrgmu.2019.078

\section{BRAIN-COMPUTER-INTERFACE TECHNOLOGY WITH MULTISENSORY FEEDBACK FOR CONTROLLED IDEOMOTOR TRAINING IN THE REHABILITATION OF STROKE PATIENTS}

Bushkova YuV' ${ }^{1}$, Ivanova GE¹, Stakhovskaya $\mathrm{LV}^{2}$, Frolov $A A^{3}$

${ }^{1}$ Research Center of Cerebrovascular Pathology and Stroke, Ministry of Health of the Russian Federation, Moscow, Russia

2 Pirogov Russian National Research Medical University, Moscow, Russia

${ }^{3}$ Institute of Higher Nervous Activity and Neurophysiology, Russian Academy of Sciences, Moscow, Russia

Motor recovery of the upper limb is a priority in the neurorehabilitation of stroke patients. Advances in the brain-computer interface (BCl) technology have significantly improved the quality of rehabilitation. The aim of this study was to explore the factors affecting the recovery of the upper limb in stroke patients undergoing $\mathrm{BCl}$ based rehabilitation with the robotic hand. The study recruited 24 patients (14 men and 10 women) aged 51 to 62 years with a solitary supratentorial stroke lesion. The lesion was left-hemispheric in 11 (45.6\%) patients and right-hemispheric in 13 (54.4\%) patients. Time elapsed from stroke was 4.0 months (3.0; 12.0$)$. The median MoCa score was 25.0 (23.0; 27.0). The rehabilitation course consisted of 9.5 sessions (8.0; 10.0). We established a significant moderate correlation between motor imagery performance (the MIQ-RS score) and the efficacy of patient-BCl interaction. Patients with high MIQ-RS scores (47.5 (32.0; 54.0 ) achieved a better control of the BCl-driven hand exoskeleton (63.0 (54.0; 67.0), $R=0.67 ; p<0.05)$. Recovery dynamics were more pronounced in patients with high MIQ-RS scores: the median score on the Fugl-Meyer Assessment scale was $14(8.0 ; 16.0)$ points vs $10(6.0 ; 13.0)$ points in patients with low MIQ-RS scores. However, the difference was not significant. Thus, we established a correlation between a patient's ability for motor imagery (MIQ-RS) and the efficacy of patient-BCl interaction A larger patient sample might be necessary to assess the effect of these factors on motor recovery dynamics.

Keywords: stroke, neurorehabilitation, brain-computer interface, ideomotor training

Funding: the study was supported by the Russian Foundation for Basic Research (Grant ID 16-29-08247 ofi_m).

Author contribution: Bushkova YuV — study planning; literature analysis; data acquisition, analysis and interpretation; manuscript preparation; Ivanova GE — study planning; literature analysis; data interpretation; manuscript preparation; Stakhovskaya LV — study planning; data interpretation; manuscript preparation; Frolov AA - manuscript preparation.

Compliance with ethical standards: the study was approved by the Ethics Committee of the Research Center of Neurology (Protocol № 12/14 dated December 10, 2014). The iMove study has been registered on Clinical Trials. gov (identifier: NCT02325947). All patients gave informed consent.

$\triangle$ Correspondence should be addressed: Yuliya V. Bushkova

Ostrovityanova, 1, str. 10, Moscow, 117342; Julijabush777@gmail.com

Received: 12.11.2019 Accepted: 24.11.2019 Published online: 07.12.2019

DOI: 10.24075/brsmu.2019.078 
Инсульт является одной из основных причин, определяющих длительную инвалидизацию пациентов во всем мире. Двигательный и когнитивный десицит, возникающий вследствие инсульта, отрицательно влияет на мобильность пациентов, значительно ограничивает возможности в повседневной бытовой деятельности, тем самым ухудшая их социальную интеграцию и снижая вероятность возврата к профессиональной деятельности [1, 2].

Нейрореабилитация (НР), основанная на принципах системности, последовательности, специфичности, адекватности реабилитационного пособия, значимо уменьшает проявления неврологического дефицита, возникшего вследствие инсульта у данной категории больных.

В основе НР лежит системная организация мозговых функций, которая определяет резервные возможности головного мозга в условиях патологического процесса. В процессе двигательного переобучения пациента происходит реорганизация его мозговых функций, что в итоге приводит к восстановлению или компенсации нарушенных функций. Главной целью НР является достижение истинного восстановления. Такой подход представляется наиболее правильным и заключается в том, что функции организма полностью возвращаются или максимально приближаются к исходному состоянию. Восстановление утраченных функций большей частью происходит за счет перестройки сохранных функциональных систем. Этот процесс обеспечивают пластичность нервной системы и анатомические связи между ее отделами.

Реабилитация неврологических пациентов в настоящее время располагает большим количеством методик, которые направлены на активацию пластических процессов в нейронных сетях, замещающих или компенсирующих утраченную функцию. Однако в большом числе клинических случаев восстановление утраченных функций происходит в ограниченном объеме. Развитие методики активации моторной коры, специфичной конкретному движению, а именно технологии интерфейса мозгкомпьютер-экзоскелет кисти (ИМКЭ кисти), качественно улучшило реабилитацию в этом направлении [3, 4].

ИМКЭ кисти - это устройство, использующее нейрофизиологическую активность мозга для прямого управляющего воздействия на внешние приборы без Включения типичных нейромышечных путей [5]. В основе реабилитационного тренажера ИМКЭ кисти с визуальной и кинестетической обратной связью лежит способность пациентов генерировать различные ЭЭГ-сигналы (в нашем случае $\mu$-ритм (мю-ритм) на ЭЭГ в двигательных зонахмозга), что позволяет «соединять мозг» С внешними устройствами без участия нейромышечных путей. Реабилитационный тренажер ИМКЭ использует ментальные двигательные представления (МДП) выполнения активных движений, которые, по данным функциональной нейровизуализации, активизируют двигательные зоны коры головного мозга [6], за исключением случаев, когда инсульт нарушает способность мозга формировать МДП [7].

Известно, что многократное мысленное повторение, называемое идеомоторной тренировкой (ИТ), способствует не только формированию двигательного навыка в нормальных физиологических условиях, но и восстановлению нарушенного или утраченного вусловиях патологии центральной нервной системы. Идеомоторное упражнение - это выполнение человеком того или иного активного двигательного действия мысленно (по представлению), так называемая мысленная тренировка.
Пациенту дают инструкцию представить выполнение определенного движения (например, вытянуть руку, раскрыть кисть, сжать кисть в кулак, взять со стола чашку и т. п.) от первого или третьего лица. Если заданное мысленное действие выполняется от первого лица, то с большой долей вероятности пациент выполняет его кинестетически. Если пациент выполняет идеомоторное действие от третьего лица, то такой способ выполнения соответствует визуальному [8]. Кинестетический способ решения ментальной задачи пациентами более прочно ассоциируется со способностью успешно взаимодействовать с реабилитационными нейрокомпьютерными интерфейсами по сравнению с визуальным вариантом решения ментальной задачи [9].

Существует несколько основных гипотез относительно механизма действия мысленных тренировок. Так называемая нервно-мышечная теория [10] основана на предположении, что мысленная тренировка вызывает подпороговую активацию мышц, участвующих в мысленном движении, и приводит к закреплению двигательной программы. Согласно второй теории, представление движений способствует возникновению специфических нейрофизиологических паттернов, аналогичных тем, которые происходят при условии выполнения реального движения, в проекции двигательных областей коры. Исследования с применением функциональной МРТ (fMRI) говорят о реорганизации двигательной системы в обоих полушариях головного мозга на фоне тренировок представления движения [6, 11].

Режим дозирования идеомоторных тренировок, по данным рандомизированных клинических исследований, достаточно вариабелен. Статистически значимый эффект (по двигательным шкалам FMA и ARAT) был показан при занятиях по 30 мин 2 раза в неделю в течение 6 недель [12]. При этом для ментальных тренировок были выбраны функционально значимые движения вроде достижения и захвата объекта, использования письменных принадлежностей и т. п.

Однако использование ИТ для восстановления нарушенных движений у взрослых пациентов после мозговой катастрофы дает противоречивые результаты [13]. Нарушения элементов контроля двигательной системы мозга негативно сказываются как на возможности выполнять движения, так и на способности к их представлению. Виной тому могут быть когнитивные нарушения, развивающиеся у больных вследствие инсульта $[1,14]$.

Перспективы применения у больных с повреждением головного мозга реабилитационных технологий, основанных на представлении движения, дают обнадеживающие результаты [12]. ИТ рассматривают в виде дополнительного метода в составе комплексной реабилитационной терапии. Сочетание идеомоторного тренинга с базовой лечебной физкультурой, соответствующей неврологическому дефициту, лежит в основе целеориентированной терапии (англ. repetitive taskspecific practice, RTP). Ранее было показано, что эффект тренировок с представлением движений на нейронную пластичность развивается и сохраняется в долгосрочной перспективе, например, при систематическом многодневном графике мысленных тренировок [15].

Представление движения субъективно, в связи с этим имеются сложности по объективизации этого процесса. Разные исследователи предлагали идеи использования реакции десинхронизации сенсомоторных ритмов ЭЭГ, возникающих на фоне представления движения $[4,16]$. Десинхронизация сенсомоторных ритмов ЭЭГ 
действительно позволяет детектировать мысленное представление движения, однако специсичность такой реакции остается не до конца изученной.

В литературе было уделено внимание необходимости оценки индивидуальной способности к представлению движений [17]. Они описали тест анкетного типа - MIQ (англ. movement imagery questionnaire), который впоследствии был доработан для применения на клинической популяции. Тесты MIQ, MIQ-R в основном применяют у спортсменов, а опросники MIQ-RS у людей, испытывающих затруднения в выполнении движений.

Реабилитационную технологию ИМКЭ кисти с мультисенсорной обратной связью (визуальной и кинестетической) можно обозначить как контролируемый идеомоторный тренинг. В основе ИТ, контролируемой посредством ИМКЭ кисти, лежит усиление афферентной стимуляции с верхней конечности посредством механической работы экзоскелета, только в ответ на успешное решение пациентом предъявленной ему ментальной задачи.

Таким образом, одной из важнейших проблем при изучении представления движений является определение способности пациента к реализации ментальной двигательной программы, так как именно это определяет эфффективность взаимодействия пациента с данной реабилитационной технологией.

Целью нашего исследования было изучить факторы, влияющие на двигательное восстановление верхней конечности у пациентов после инсульта в раннем восстановительном периоде на фоне применения технологии ИМКЭ.

\section{ПАЦИЕНТЫ И МЕТОДЫ}

Исследование проводили в Научно-исследовательском институте цереброваскулярной патологии и инсульта РНИМУ им Н. И. Пирогова на базе ГКБ 31 г. Москвы, неврологического отделения для больных с инсультом в период с сентября 2018 по апрель 2019 г.

Критерии включения пациентов в исследование: мужчины и женщины в возрасте 18-80 лет; наличие в головном мозге единичного очага инсультной этиологии подкорковой локализации давностью до двух лет; сохранность когнитивных функций, равная не менее 22 баллов по Монреальской шкале оценки когнитивных функций (MoCA) [18]; двигательный дефицит в верхней конечности от 0 до 4 баллов по Британской шкале оценки мышечной силы (MRCMS) [19]; праворукость пациента по данным Эдинбургского опросника мануальной асимметрии [20].

Критерии исключения: давность инсульта более двух лет; леворукость по данным Эдинбургского опросника мануальной асимметрии; выраженное снижение когнитивных функций; сенсорная афазия; грубая моторная афазия; грубое нарушение зрения, не позволяющее выполнять визуально предъявляемые на экране компьютера инструкции; наличие мышечной контрактуры в руке, достигающей 4 баллов по модифицированной шкале Ashworth (mAS) [21].

В текущий анализ были включены данные 24 пациентов, из которых было 14 (58,3\%) мужчин и 10 (41,7\%) женщин. Медиана возраста составила 56,5 (51,0; 62,0) лет. Единичный очаг инсультной этиологии ишемического характера - у 20 (83,3\%) пациентов и геморрагического характера - у 4 (16,7\%) пациентов, супратенториальной локализации - у всех 24 (100\%) пациентов (по данным
KT, МРT). Левополушарное поражение выявлено в 11 (45,6\%) случаях, правополушарное - в 13 (54,4\%) случаях. Давность инсульта - 4 (3,0;12,0) месяца. Медиана МоСА составила 25,0 (23,0; 27,0) баллов, что соответствует наличию умеренного когнитивного десицита. Часть пациентов, участвовавших в исследовании, получали терапию в условиях стационара $(n=11)$, остальные амбулаторно $(n=13)$. Курс занятий на тренажере включал 9,5 (8,0; 10,0) процедур.

В исследовании использовали ИМКЭ кисти с кинестетической и визуальной обратной связью («Экзокисть-2») (РНИМУ им. Н. И. Пирогова; Москва) (см. рис.).

ИМКЭ кисти основан на анализе паттернов ЭЭГ и распознавании реакции синхронизации/десинхронизации сенсомоторного н-ритма при воображении движений рук. Сигналы ЭЭГ фильтровали в полосе частот 5-30 Гц. В исследовании применяли классисикатор паттернов ЭЭГ, основанный на методе Байеса [22]. В качестве показателей точности классификации использовали индекс каппа Коэна (при идеальном распознавании к = 1 , при случайном распознавании к = 0 [23]) и процент правильных ответов классификатора (более $33 \%$ - распознавание выше случайного, так как пациенты выполняют по инструкции ментальные задачи).

Результаты распознавания ментальной задачи предъявляли пациенту по зрительной и кинестетической обратной связи: в случае успешного распознавания классисикатором задачи, соответствующей предъявляемой инструкции, метка в середине экрана принимала зеленый цвет, а экзоскелет разгибал пальцы кисти. При распознавании других задач, цвет метки не менялся, а экзоскелет не срабатывал [4]. Таким образом, этот вид терапии можно назвать управляемой идеомоторной

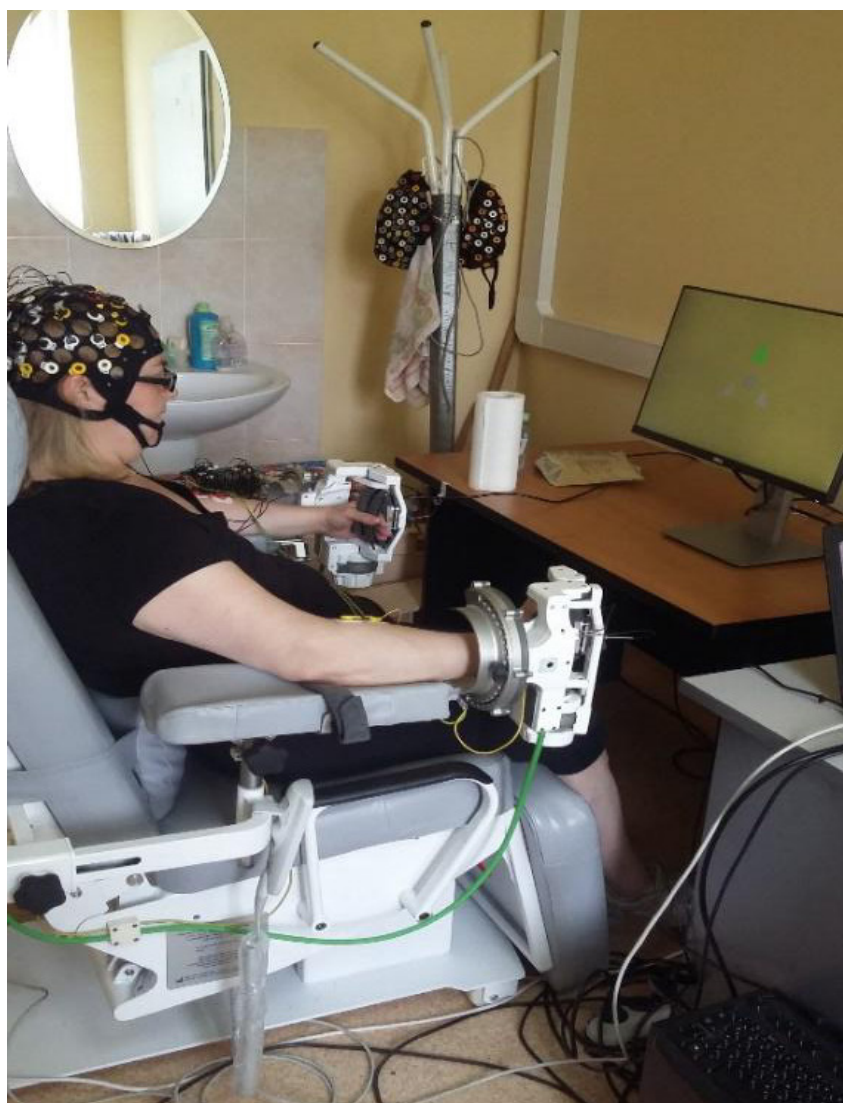

Рис. Внешний вид ИМКЭ кисти с кинестетической и визуальной обратной связью («Экзокисть-2») 
тренировкой с использованием принципов многоканальной биологической обратной связи по зрительному, кинестетическому анализаторам и ЭЭГ.

Во время процедуры на голову пациента надевали шапочку с электродами для регистрации ЭЭГ, под каждый из которых был нанесен электродный гель. Кисти обеих рук фиксировали электромеханизированным экзоскелетом, предназначенным для разгибания пальцев кисти в физиологическом объеме. В процессе тренинга человек сидел в кресле перед компьютерным монитором. В центре темного экрана монитора находился круг, служащий для фиксации взгляда, вокруг него расположены три стрелки для обозначения инструкций изменяющимся цветом. Пациент выполнял одну из трех инструкций: расслабиться, кинестетически представить медленное разгибание пальцев кисти правой или левой руки. Инструкции на воображение разгибания пальцев правой или левой руки (изменение цвета стрелки справа или слева соответственно) предъявляли в случайном порядке в течение 10 с. Между инструкциями по воображению движения предъявляли инструкцию расслабиться (загоралась верхняя стрелка) тоже в течение 10 с. По инструкции «расслабиться» пациент должен спокойно сидеть и смотреть в центр экрана. Одна процедура содержала до трех вышеописанных сессий, каждая длительностью 10 мин. Между сессиями пациент отдыхал не менее 3 мин. C каждым пациентом занятия на тренажере ИМКЭ кисти проводили в течение 14-18 дней, с возможными промежутками между занятиями до 2 дней.

\section{РЕЗУЛЬТАТЫ ИССЛЕДОВАНИЯ}

Пациенты прошли курс комплексной двигательной реабилитации, включавший в себя стандартизированную методику реабилитации больных после инсульта (ООКТ 1-4 ступень, PNF, тренировки на кардиотренажере «Motomed») [24] с включением в программу занятий на тренажере ИМКЭ кисти в количестве 9,5 (8,0; 10,0) процедур.

Для оценки двигательной функции верхней конечности пациентов применяли шкалы Fugl-Meyer Assessment (FMA) [25], Британскую шкалу оценки мышечной силы (MRCWS), модифицированную шкалу спастичности Ashworth (mAS). Для оценки функциональной активности верхней конечности мы использовали Action Research Arm Test (ARAT) [26]. Для оценки повседневной жизнедеятельности применяли Barhtel Index (BI). Для оценки качества идеомоторного представления мы использовали шкалу Movement Imagery Questionnaire (MIQ-RS) для клинической популяции.

Статистическую обработку результатов проводили с помощью критериев Манна-Уитни, Уилкоксона, коэффициента корреляции Спирмена на персональном компьютере с применением пакета прикладных программ
Statistica v 13.0 (StatSoft; США). Данные представлены в виде медианы и межквартинтельного интервала (25; 75\%). Статистически значимыми считали различия при $p<0,05$.

Медиана качества управления пациентами нейрокомпьютерным интерфейсом (индекс каппа Коэна и процент распознавания) составила 58,5\% $(45,7 ; 62,6)$ ( $<$ < 0,05), что подтверждает успешность взаимодействия пациентов с нейроинтерфейсом.

Для изучения возможности и качества кинестетической компоненты идеомоторного представления на основании данных теста MIQ-RS мы разделили пациентов на две группы в зависимости от латерализации очага поражения в головном мозге (табл. 1).

Различия полученных показателей были статистически значимы. Больные с левополушарной локализацией очага инсульта оказались более успешными в формировании ментального кинестетического двигательного представления, чем пациенты с правополушарной локализацией очага, что не противоречит данным литературы [27].

Однако попытка поиска корреляции между качеством моторного представления больными в зависимости от «стороны инсульта» и эффективностью взаимодействия с ИМКЭ кисти, по данным классификатора, успеха не принесла. В связи с этим пациенты были разделены на две группы по качеству моторного представления, исходя из данных шкалы MIQ-RS, в зависимости от полученных баллов. Первую группу составили те из пациентов, кто набрал более 50\% баллов в субшкале от максимального значения, отражающей кинестетическую составляющую представления; вторую группу — те, кто набрал менее 50\%. 13 пациентов субъективно оценили возможности своего кинестетического представления достаточно высоко, медиана показателя составила 47,5 (32,0; 54,0) балла. Медиана показателя остальных 11 пациентов - 27,0 (15,0; 29,0) баллов. Различие в группах по выделенному показателю достигло статистической значимости $(p<0,05)$. Далее была изучена связь качества моторного представления пациентами с успешностью взаимодействия их с ИМКЭ кисти (табл. 2).

При анализе полученных данных была выявлена значимая умеренная корреляция между успешностью моторного представления (MIQ-RS) и эффективностью взаимодействия пациентов с ИМКЭ кисти. Другими словами, пациенты с высокими показателями по шкале MIQ-RS показывали достоверно высокие значения классификатора, а пациенты с более низкими взаимодействовали с ИМКЭ кисти достоверно хуже.

Зависимость восстановления двигательной и функциональной активности пациентов от эффективности их взаимодействия с ИМКЭ кисти представлена в табл. 3.

В обеих группах в восстановлении двигательной и функциональной активности верхней конечности была

Таблица 1. Качество моторного представления пациентами в зависимости от латерализации очага поражения в головном мозге

\begin{tabular}{|c|c|c|}
\hline Показатель/Критерий Манна-Уитни & Правополушарная локализация очага $(n=13)$ & Левополушарная локализация очага $(n=11)$ \\
\hline $\mathrm{MIQ}-\mathrm{RS}(p<0,05)$ & $29(18,0 ; 35,0)$ & $44,0(25,0 ; 54,0)$ \\
\hline
\end{tabular}

Таблица 2. Данные эффективности взаимодействия пациентов с ИМКЭ кисти в зависимости от качества моторного представления пациентов по данным МIQ-RS

\begin{tabular}{|l|c|c|}
\hline \multicolumn{1}{|c|}{ Показатель } & Пациенты с MIQ-RS $>50 \%(n=13)$ & Пациенты с MIQ-RS < 50\% $(n=11)$ \\
\hline Коэффициент корреляции Спирмена & $R=0,67(p<0,05)$ & $R=0,43(p<0,05)$ \\
\hline MIQ-RS & $47,5(32,0 ; 54,0)$ & $27,0(15,0 ; 29,0)$ \\
\hline Классификатор, $p<0,05$ & $63,0(54,0 ; 67,0)^{\star}$ & $39,0(32,0 ; 48,0)^{\star}$ \\
\hline
\end{tabular}

Примечание: * $-p<0,05$. 
Таблица 3. Зависимость восстановления двигательной, функциональной активности верхней конечности и бытовой активности пациентов от эффективности их взаимодействия с ИМКЭ кисти

\begin{tabular}{|l|c|c|c|}
\hline \multicolumn{1}{|c|}{ Показатель/Критерий Манна-Уитни } & Пациенты с MIQ-RS $>50 \%(n=13)$ & \multicolumn{2}{|c|}{ Пациенты c MIQ-RS < 50\% $(n=11)$} \\
\hline \multirow{2}{*}{ Классификатор, $p<0,05$} & \multicolumn{2}{|c|}{$63,0(54,0 ; 67,0)$} & \multicolumn{2}{|c|}{$39,0(32,5 ; 48,5)$} \\
\hline & До & После & \multicolumn{2}{|c|}{ После } \\
\hline FMA общий балл & $88,0(62,0 ; 102,0)$ & $102,0(66,0 ; 112,0)^{\star}$ & $95,5(67,0 ; 109,0)$ \\
\hline MRCWS & $3,0(2,0 ; 4,0)$ & $3,0(3,0 ; 4,0)$ & $3,0(2,0 ; 4,0)$ \\
\hline mAS & $1,5(3,0 ; 1,0)$ & $1,0(1,0 ; 1,0)^{\star}$ & $2,0(1,0 ; 2,0)$ \\
\hline ARAT общий балл & $37,0(4,0 ; 47,0)$ & $42,0(6,0 ; 53,0)^{\star}$ & $35,0(5,0 ; 43,0)$ \\
\hline BI & $90,0(75,0 ; 95,0)$ & $95,0(80,0 ; 100,0)^{\star}$ & $87,5(75,0 ; 100,0)$ \\
\hline
\end{tabular}

выявлена положительная статистически значимая динамика. У пациентов с более успешным представлением движения динамика была более выраженной, но не достигла значимых различий с пациентами другой группы. Кроме того, на данной выборке пациентов не был обнаружено значимой корреляции между успешностью взаимодействия пациентов с нейроинтерфейсом (по данным классификатора) и выраженностью динамики двигательного восстановления функции верхней конечности, что возможно объясняется недостаточным количеством участников. Положительную динамику по шкале ВІ наблюдали у пациентов обеих групп за счет движений, требующих бимануального выполнения.

\section{ОБСУЖДЕНИЕ РЕЗУЛЬТАТОВ}

Неинвазивные нейроинтерфейсы позволяют людям контролировать внешние устройства с помощью декодирования мозговой активности в режиме реального времени. В неинвазивных подходах ИМК пользователи участвуют в когнитивной задаче, такой как визуальное внимание или двигательное представление, в то время как сигналы их мозга записывают с помощью электроэнцефалографии (ЭЭГ) и декодируют в режиме онлайн для окончательного контроля над внешними устройствами [28]. Несмотря на технические достижения и продолжающееся усовершенствование технологии нейроинтерфейсов, способность эффективно взаимодействовать остается ограниченной для некоторых пользователей здоровой популяции. Показано, что доля пользователей, которые не могут добиться успешного контроля, находится в диапазоне 15-30\% [29]. По результатам исследований с участием большего количества субъектов, до 50\% пользователей не могут достичь точности выше 70\% - порога, который обычно считают более точным для определения успешного контроля [30]. Важно, что эти показатели отражают возможности лиц здоровой популяции. В нашем исследовании участвовали пациенты неврологического профиля, имеющие умеренный когнитивный десицит. Несмотря на это часть пациентов во взаимодействии с нейроинтерфейсом были более успешными: индекс каппа Коэна и процент распознавания у них составили 63,0\% (54,0; 67,0), а часть пациентов менее успешными - их показатель составил 39,0\% $(32,0 ; 48,0)$. Уровень полученных данных коррелировал с результатами тестирования пациентов с помощью шкаль MIQ-RS. Результат работы имеет большое теоретическое и практическое значение, так как наглядно подтверждает единство копнитивных и двигательных процессов в организме человека, демонстрирует необходимость дуалистического подхода к процессу восстановления как двигательных, так и когнитивных функций при поражении мозга, ставит задачи по разработке персонифицированного подхода к использованию технологии в целях получения наилучшего результата, что, в свою очередь, приведет к более рациональному использованию реабилитационных ресурсов.

\section{ВЫВОДЫ}

В исследовании выявлена взаимосвязь способности пациентов к моторному представлению (по данным шкалы MIQ-RS) с эфффективностью взаимодействия пациентов с нейроинтерфейсом. Для выявления влияния успешности взаимодействия пациентов с нейроинтерфейсом (по данным классификатора) на динамику двигательного восстановления функции верхней конечности вероятно требуется продолжить исследование с увеличенным объемом выборки.

\section{Литература}

1. Куташов В. А., Будневский А. В., Припутневич Д. Н., Суржко Г. В. Психологические особенности пациентов с последствиями кровообращения, затрудняющими социальную адаптацию. Вестник неврологии, психиатрии и нейрохирургии. 2014; (8): 8-13.

2. Roman GC. Facts, myths, and controversies in vascular dementia. J Neurol Sci. 2004; 226: 49-52.

3. Ang KK, Phua KS, Wang C, Chin ZY, Kuah CW, Low W, et al. Randomized Controlled Trial of EEG-Based Motor Imagery BrainComputer Interface Robotic Rehabilitation for Stroke. Clinical EEG and neuroscience. 2015; 46 (4): 310-20. https://doi. org/10.1177/1550059414522229

4. Фролов А. А., Мокиенко О. А., Люкманов Р. Х., Черникова Л. А., Котов С. В. и др. Предварительные результаты контролируемого исследования эффективности технологии ИМК-экзоскелет при постинсультном парезе руки. Вестник РГМУ. 2016; (2): 175. 5. Novak DA, Grefkes C, Arneli M, Fink GR. Interhemispheric competition after stroke: brain stimulation to enhance recovery of function of the affected hand. Neururehabil Neural Repair. 2009; (23): 641-57.

6. Sharma N, Simmons LH, Jones PS, Day DJ, Carpenter TA, Pomeroy VM, et al. Motor imagery after subcortical stroke: a functional magnetic resonance imaging study. Stroke. 2009; 40 (4): 315-24.

7. Johnson SH, Sprehn G, Saykin AJ. Intact motorimagery in chronic upper limb hemiplegics: evidence foractivity-independent action representations. Journal of Cognitive Neuroscience. 2002; 14 (6): 841-52.

8. Barclay-Goddard RE, Stevenson TJ, Poluha W, Thalman L. Mental practice for treating upper extremity deficits in individuals with 
hemiparesis after stroke. Cochrane Database Syst Rev. 2011; 11 (5): CD005950. DOI: 10.1002/14651858.CD005950.pub4.

9. Chholak P, Niso G, Maksimenko VA, Kurkin SA, Frolov NS, Pitsik EN et al. Visual and kinesthetic modes affect motor imagery classification in untrained subjects. 2019 Jul 8; 9 (1): 9838. DOI:10.1038/s41598-019-46310-9.

10. Schmidt R, Lee T. Motor Control and Learning: A Behavioral Emphasis. Champaign, IL: Human Kinetics, 1999; 592 p.

11. Bajaj S, Butler AJ, Drake D, Dhamala M. Brain effective connectivity during motor-imagery and execution following stroke and rehabilitation. Neuroimage Clin. 2015 (8): 572-82. https://doi. org/10.1016/j.nicl.2015.06.006.

12. Page SJ, Levine P, Leonard A. Mental practice in chronic stroke: results of a randomized, placebo-controlled trial. Stroke. 2007 Apr; 38 (4): 1293-7.

13. Braun SM, Beurskens AJ, Borm PJ, Schack T, Wade DT. The effects of mental practice in stroke rehabilitation: a systematic review. Arch Phys Med Rehabil. 2006 Jun; 87 (6): 842-52.

14. Román GC. Facts, myths, and controversies in vascular dementia. J Neurol Sci. 2004 Nov 15; 226 (1-2): 49-52.

15. Sauvage C, De Greef N, Manto M, Jissendi P, Nioche C, Habas C. Reorganization of large-scale cognitive networks during automation of imagination of a complex sequential movement // Journal of neuroradiology. 2015; 42 (2): 115-25.

16. Каплан А. Я. Нейрофизиологические основания и практические реализации технологии мозг машинных интерфейсов в неврологической реабилитации. Физиология человека. 2016; 42 (1): 118-27.

17. Hall CR, Martin KA. Measuring movement imagery abilities: A revision of the Movement Imagery Questionnaire. Journal of mental imagery. 1997

18. Bocti C, Legault V, Leblanc N, Berger L, Nasreddine Z, BeaulieuBoire I, et al. Vascular cognitive impairment: most usefu subtests of the Montreal Cognitive Assessment in minor stroke and transient ischemic attack. Dement Geriatr Cogn Disord. 2013; 36 (3-4): 154-62.

19. Compston A. Aids to the investigation of peripheral nerve injuries.
Medical Research Council: Nerve Injuries Research Committee. His Majesty's Stationery Office: 1942; pp. 48 (iii) and 74 figures and 7 diagrams; with aids to the examination of the peripheral nervous system. By Michael O'Brien for the Guarantors of Brain. Saunders Elsevier, 2010; pp. 64.

20. Oldfield RC. The assessment and analysis of handedness: the Edinburgh inventory. Neuropsychologia. 1971 Mar; 9 (1): 97-113.

21. Bohannon RW, Smith MB. Interrater reliability of a modified Ashworth scale of muscle spasticity. Phys Ther. 1987 Feb; 67 (2): 206-7.

22. Бобров П. Д., Коршаков А. В., Рощин В. Ю., Фролов А. А. Байесовский подход $\mathrm{k}$ реализации интерфейса мозгкомпьютер, основанного на представлении движений. Журнал высшей нервной деятельности. 2012; 62 (1): 89-99.

23. Jorgensen HS, Nakayama H, Raaschou HO, Olsen TS. Recovery of walking function in stroke patients: the Copenhagen Stroke Study. Archives of Physical Medicine and Rehabilitation. 1995. 76 (1): 27-32.

24. Инсульт у взрослых: центральный парез верхней конечности Клинические рекомендации МКБ10: 160/161/162/163/164/169.

25. Sanford J, Moreland J, Swanson LR, Stratford PW, Gowland C. Reliability of the Fugl-Meyer assessment for testing motor performance in patients following stroke. Phys Ther. $1993 \mathrm{Jul}$; 73 (7): 447-54

26. Doussoulin SA, Rivas SR, Campos SV. Validation of «Action Research Arm Test» (ARAT) in Chilean patients with a paretic upper limb after a stroke. Rev Med Chil. 2012 Jan; 140 (1): 59-65. Spanish.

27. Боголюбов В. М. Медицинская реабилитация. М.: БИНОМ, 2010; c. 21.

28. Wolpaw JR, McFarland DJ. Control of a two-dimensional movement signal by a noninvasive brain-computer interface in humans. Proc Natl Acad Sci USA. 2004 (101): 17849-54.

29. Vidaurre $\mathrm{C}$, Blankertz B. Towards a cure for BCl illiteracy. Brain Topogr. 2010; (23): 194-8.

30. Ahn M, Chan S. Performance variation in motor imagery brain computer interface: A brief review. J Neurosci Methods. 2015; (243): 103-10.

\section{References}

1. Kutashov VA, Budnevskiy AV, Priputnevich DN, Surzhko GV. Psychological features of patients with consequences of acute disorders of cerebral circulation, hamper their social adaptation. Vestnik nevrologii, psihiatrii i nejrohirurgii. 2014; (8): 8-13.

2. Roman GC. Facts, myths, and controversies in vascular dementia. J Neurol Sci. 2004; 226: 49-52.

3. Ang KK, Phua KS, Wang C, Chin ZY, Kuah CW, Low W, et al, Randomized Controlled Trial of EEG-Based Motor Imagery BrainComputer Interface Robotic Rehabilitation for Stroke. Clinical EEG and neuroscience. 2015; 46 (4): 310-20. https://doi. org/10.1177/1550059414522229.

4. Frolov AA, Mokienko OA, Lyukmanov RKh, Chernikova LA, Kotov SV, et al. Preliminary results of a controlled study of the effectiveness of IMC-exoskeleton technology for post-stroke paresis of the arm. Vestnik RSMU. 2016; (2): 175.

5. Novak DA, Grefkes C, Arneli M, Fink GR. Interhemispheric competition after stroke: brain stimulation to enhance recovery of function of the affected hand. Neururehabil Neural Repair. 2009; (23): 641-57.

6. Sharma N, Simmons LH, Jones PS, Day DJ, Carpenter TA, Pomeroy VM, et al. Motor imagery after subcortical stroke: a functional magnetic resonance imaging study. Stroke. 2009; 40 (4): 315-24.

7. Johnson SH, Sprehn G, Saykin AJ. Intact motorimagery in chronic upper limb hemiplegics: evidence foractivity-independent action representations. Journal of Cognitive Neuroscience. 2002; 14 (6): 841-52.

8. Barclay-Goddard RE, Stevenson TJ, Poluha W, Thalman L. Mental practice for treating upper extremity deficits in individuals with hemiparesis after stroke. Cochrane Database Syst Rev. 2011; 11 (5): CD005950. DOI: 10.1002/14651858.CD005950.pub4.

9. Chholak P, Niso G, Maksimenko VA, Kurkin SA, Frolov NS, Pitsik EN et al. Visual and kinesthetic modes affect motor imagery classification in untrained subjects. 2019 Jul 8; 9 (1): 9838. DOI:10.1038/s41598-019-46310-9.

10. Schmidt R, Lee T. Motor Control and Learning: A Behaviora Emphasis. Champaign, IL: Human Kinetics, 1999; 592 p.

11. Bajaj S, Butler AJ, Drake D, Dhamala M. Brain effective connectivity during motor-imagery and execution following stroke and rehabilitation. Neuroimage Clin. 2015 (8): 572-82. https://doi. org/10.1016/j.nicl.2015.06.006.

12. Page SJ, Levine P, Leonard A. Mental practice in chronic stroke: results of a randomized, placebo-controlled trial. Stroke. 2007 Apr; 38 (4): 1293-7.

13. Braun SM, Beurskens AJ, Borm PJ, Schack T, Wade DT. The effects of mental practice in stroke rehabilitation: a systematic review. Arch Phys Med Rehabil. 2006 Jun; 87 (6): 842-52.

14. Román GC. Facts, myths, and controversies in vascular dementia. J Neurol Sci. 2004 Nov 15; 226 (1-2): 49-52.

15. Sauvage C, De Greef N, Manto M, Jissendi P, Nioche C, Habas C. Reorganization of large-scale cognitive networks during automation of imagination of a complex sequential movement // Journal of neuroradiology. 2015; 42 (2): 115-25.

16. Kaplan AYa. Neurophysiological foundations and practical implementation of the technology of the brain of machine interfaces in neurological rehabilitation. Human Physiology. 2016; 42 (1): 118-27.

17. Hall CR, Martin KA. Measuring movement imagery abilities: A revision of the Movement Imagery Questionnaire. Journal of mental imagery. 1997.

18. Bocti C, Legault V, Leblanc N, Berger L, Nasreddine Z, BeaulieuBoire I, et al. Vascular cognitive impairment: most usefu 
subtests of the Montreal Cognitive Assessment in minor stroke and transient ischemic attack. Dement Geriatr Cogn Disord. 2013; 36 (3-4): 154-62.

19. Compston A. Aids to the investigation of peripheral nerve injuries. Medical Research Council: Nerve Injuries Research Committee. His Majesty's Stationery Office: 1942; pp. 48 (iii) and 74 figures and 7 diagrams; with aids to the examination of the peripheral nervous system. By Michael O'Brien for the Guarantors of Brain. Saunders Elsevier, 2010; pp. 64.

20. Oldfield RC. The assessment and analysis of handedness: the Edinburgh inventory. Neuropsychologia. 1971 Mar; 9 (1): 97-113.

21. Bohannon RW, Smith MB. Interrater reliability of a modified Ashworth scale of muscle spasticity. Phys Ther. 1987 Feb; 67 (2): 206-7.

22. Bobrov PD, Korshakov AV, Roshchin VYu, Frolov AA. Bayesian approach to the implementation of the brain-computer interface based on the representation of movements. Journal of Higher Nervous Activities. 2012; 62 (1): 89-99.

23. Jorgensen HS, Nakayama H, Raaschou HO, Olsen TS. Recovery of walking function in stroke patients: the Copenhagen Stroke
Study. Archives of Physical Medicine and Rehabilitation. 1995. 76 (1): 27-32.

24. Stroke in adults: central paresis of the upper limb. Clinical recommendations ICD10: 160/161/162/163/164/169.

25. Sanford J, Moreland J, Swanson LR, Stratford PW, Gowland C. Reliability of the Fugl-Meyer assessment for testing motor performance in patients following stroke. Phys Ther. 1993 Jul; 73 (7): 447-54

26. Doussoulin SA, Rivas SR, Campos SV. Validation of «Action Research Arm Test» (ARAT) in Chilean patients with a paretic upper limb after a stroke. Rev Med Chil. 2012 Jan; 140 (1): 59-65. Spanish.

27. Bogolyubov VM. Medicinskaja reabilitacija. M.: BINOM, 2010; s. 21

28. Wolpaw JR, McFarland DJ. Control of a two-dimensional movement signal by a noninvasive brain-computer interface in humans. Proc Natl Acad Sci USA. 2004 (101): 17849-54.

29. Vidaurre $\mathrm{C}$, Blankertz B. Towards a cure for BCl illiteracy. Brain Topogr. 2010; (23): 194-8.

30. Ahn M, Chan S. Performance variation in motor imagery brain computer interface: A brief review. J Neurosci Methods. 2015; (243): 103-10. 